\title{
Incremento de la Eficiencia en Centrales Termoeléctricas por Aprovechamiento de los Gases de la Combustión
}

Jesús M. Blanco y Francisco Peña

Universidad del País Vasco / E.H.U., Escuela Técnica Superior de Ingeniería, Alda. Urquijo s/n, 48013 Bilbao-España (e-mail: jesusmaria.blanco@ehu.es)

Recibido Nov. 18, 2010; Aceptado Ene. 20, 2011; Versión Final recibida Feb. 21, 2011

\section{Resumen}

Se presenta una propuesta para aprovechar el calor latente del vapor condensado en un economizador usado para precalentar el agua de alimentación al generador de vapor, con el fin de mejorar la eficiencia de las centrales termoeléctricas. Se expone una metodología novedosa aplicada a centrales de más de $350 \mathrm{MW}$ de potencia y que queman gas natural. Se presenta también un detallado estudio de las distintas alternativas propuestas, evaluando finalmente el efecto directo sobre su viabilidad económica. Se han llegado a obtener incrementos de más del $0.6 \%$ en la eficiencia energética global, además de cortos períodos de retorno simple de la inversión dependiendo del número de horas anuales efectivas de funcionamiento de la central.

\section{Increasing Thermal Efficiency at Thermoelectric Power Plants Through Combustion Exhaust Gases Management}

\begin{abstract}
This paper presents a proposal for using the latent heat of the steam condensed in an economizer used to preheat the feeding water to the steam generator, with the aim of increasing the efficiency of thermal power plants. A novel methodology applied to power plants producing over $350 \mathrm{MW}$ power plants and that use natural gas is developed. Also, a detailed study of the different alternatives proposed is presented and their direct effects on the economic feasibility have been evaluated. Increases up to $0.6 \%$ in the global efficiency are obtained, besides short return investment periods, depending on the annual effective working hours of the power plant.
\end{abstract}

Keywords: thermoelectric power plants, global efficiency, exhaust gases, latent heat 


\section{INTRODUCCIÓN}

En su forma más clásica, las centrales termoeléctricas queman combustible en un generador de vapor para producir vapor el cual se expansiona a continuación en una turbina de vapor que impulsa un alternador. Finalmente el vapor es enfriado en un Condensador. Las centrales térmicas que usan combustibles fósiles liberan a la atmósfera dióxido de carbono $\left(\mathrm{CO}_{2}\right)$, considerado el principal gas responsable del calentamiento global. También, dependiendo del combustible utilizado, pueden emitir otros contaminantes como óxidos de azufre, óxidos de nitrógeno, partículas sólidas (polvo) y cantidades variables de residuos sólidos (Blanco y Peña, 2006).

En las centrales termoeléctricas denominadas de ciclo combinado se usan los gases de la combustión del gas natural para mover una turbina de gas, y los gases residuales se aprovechan en una caldera de recuperación para generar vapor que se introduce posteriormente en una turbina de vapor. Hoy en día, la tendencia es evolucionar a instalaciones de producción de energía eléctrica más eficientes debido a la necesidad de optimizar tanto los ciclos de producción (Remiro y Lozano, 2007), como los aspectos medioambientales asociados (Demirbas, 2008). En este sentido, el gas natural es un combustible limpio que no presenta problemas de rocío ácido ni de arrastre de partículas y con un porcentaje relativamente alto de $\mathrm{H}_{2}$ en la matriz del combustible (Ion et al., 2007; Schneider y Bogdan, 2007).

Esto permite disminuir la temperatura de los gases de la combustión por debajo de la temperatura de rocío físico pudiendo aprovechar el calor latente de cambio de estado del vapor de agua formado en el proceso de combustión en un intercambiador de carcasa y tubos de acero inoxidable. Habría que considerar la posibilidad de humidificar la combustión (añadiendo agua o vapor de agua) para aumentar la presión parcial del vapor en los gases con lo cual se consigue aumentar la temperatura de rocío físico de los mismos (Guerra et al., 2005) y posibilitando una mayor recuperación de este calor latente de cambio de estado, pero las cantidades de agua a manejar hacen inviable económicamente la instalación (Chacón et al., 2006; Hongbin y Pengxiu, 2010).

En las centrales de gas natural, los gases abandonan los calentadores de aire regenerativos, cuando existen, a temperaturas del orden de los $120^{\circ} \mathrm{C}$, mientras que en centrales de fuel oil reconvertidas a gas natural lo hacen a unos $150^{\circ} \mathrm{C}$ (Blanco et al., 2006). La idea es aprovechar el calor contenido en los gases por debajo de su punto de rocío físico en un economizador a condensación que realice el efecto de recalentamiento del agua de alimentación proveniente del condensador de la primera extracción de turbina, suprimiendo de esta forma el calentador $\mathrm{N}^{\circ} 1$ de la planta y expansionando el vapor utilizado en dicho calentador hasta la presión del condensador.

De esta forma se produce una mejora en el rendimiento de la planta, lo que conlleva la consiguiente mejora medioambiental (Bastidas et al., 2010), ya que para dar la misma potencia se consume menos combustible y si consumimos el mismo combustible aumentaría la potencia de la planta (Chen et al., 2010). Hay que tener en cuenta que los gases de la combustión deben abandonar la instalación a una temperatura superior, por lo menos a $75^{\circ} \mathrm{C}$, por el problema del penacho que no supone contaminación medioambiental ya que se trata de vapor de agua. Por otro lado, la disminución del contenido de vapor de agua en los gases de la combustión disminuiría la probabilidad de formación del penacho (emisión compuesta solamente de gases o de gases con partículas y aerosoles, que emerge de una chimenea) aunque no es éste el objetivo.

El objetivo es demostrar que este tipo de aprovechamiento es viable tanto técnica, como económicamente con una inversión relativamente baja y períodos de recuperación de ésta muy interesantes (Grohn et al., 2009). El calentamiento regenerativo del agua de alimentación del generador de vapor utiliza parte del vapor de turbina para tal fin. Con esta metodología, el vapor que no es usado para el calentamiento regenerativo, se utiliza directamente en la turbina de vapor, aumentando tanto la potencia de ésta como la eficiencia energética el ciclo.

Para llevar a cabo este estudio se han escogido dos centrales tipo, una central de fuel oil reconvertida a gas natural (en adelante central reconvertida) y otra originariamente diseñada para 
gas natural, (en adelante central de gas natural), de forma que para cada una de ellas se ha llevado a cabo un detallado y riguroso estudio de la utilización de la potencia térmica, se ha analizado su comportamiento al sustituir los calentadores $\mathrm{N}^{\circ} 1$ y $\mathrm{N}^{\circ} 2$ correspondientes a las dos extracciones de baja por el economizador a condensación, comprobándose que el sistema de condensado es autorregulado ya que a medida que se va calentando el agua, el salto térmico entre el agua aportada al calentador y el vapor de la extracción es menor. En el momento en el que no hubiera salto térmico no habría condensación. Por último, se han evaluado las distintas posibilidades de recuperar este calor así como el efecto que tiene en su viabilidad económica. Las características más importantes de operación de ambas centrales se resumen a continuación en la Tabla 1.

Tabla 1: Condiciones de operación de las centrales termoeléctricas de referencia.

\begin{tabular}{|c|c|c|}
\hline & Central reconvertida & Central de gas natural \\
\hline POTENCIA BRUTA (MW) & 377 & 350 \\
\hline CONSUMO COMBUSTIBLE $\left(\mathrm{Nm}^{3} / \mathrm{h}\right)$ & 90.700 & 81.000 \\
\hline GENERADOR DE VAPOR & Babcock \& Wilcox & Durr Werke \\
\hline Caudal de aire combustión (ton/h) & 1.275 & 1.280 \\
\hline Temperatura de salida gases combustión $\left({ }^{\circ} \mathrm{C}\right)$ & 147 & 120 \\
\hline Producción de vapor (ton/h) & 1.240 & 1.090 \\
\hline Temperatura del vapor sobrecalentado $\left({ }^{\circ} \mathrm{C}\right)$ & 568 & 533 \\
\hline Temperatura del vapor recalentado $\left({ }^{\circ} \mathrm{C}\right)$ & 538 & 543 \\
\hline Presión de vapor sobrecalentado $\left(\mathrm{kg} / \mathrm{cm}^{2}\right)$ & 175 & 175 \\
\hline Presión de vapor recalentado $\left(\mathrm{kg} / \mathrm{cm}^{2}\right)$ & 36 & 43 \\
\hline TURBINA / ALTERNADOR & General Electric & KWU / Siemens \\
\hline Número de extracciones & 7 & 7 \\
\hline Refrigeración alternador & Hidrógeno & Hidrógeno \\
\hline BOMBAS DE CONDENSADO & Worthington & KSB \\
\hline Número & 2 & 2 \\
\hline Potencia motor unitaria $(\mathrm{kW})$ & 766 & 1.100 \\
\hline
\end{tabular}

\section{METODOLOGÍA}

La Figura 1 muestra un esquema que ilustra la modificación que se propone para aprovechar el calor latente del vapor de agua de los gases de la combustión para ambos tipos de centrales la cual se puede observar en línea discontinua.

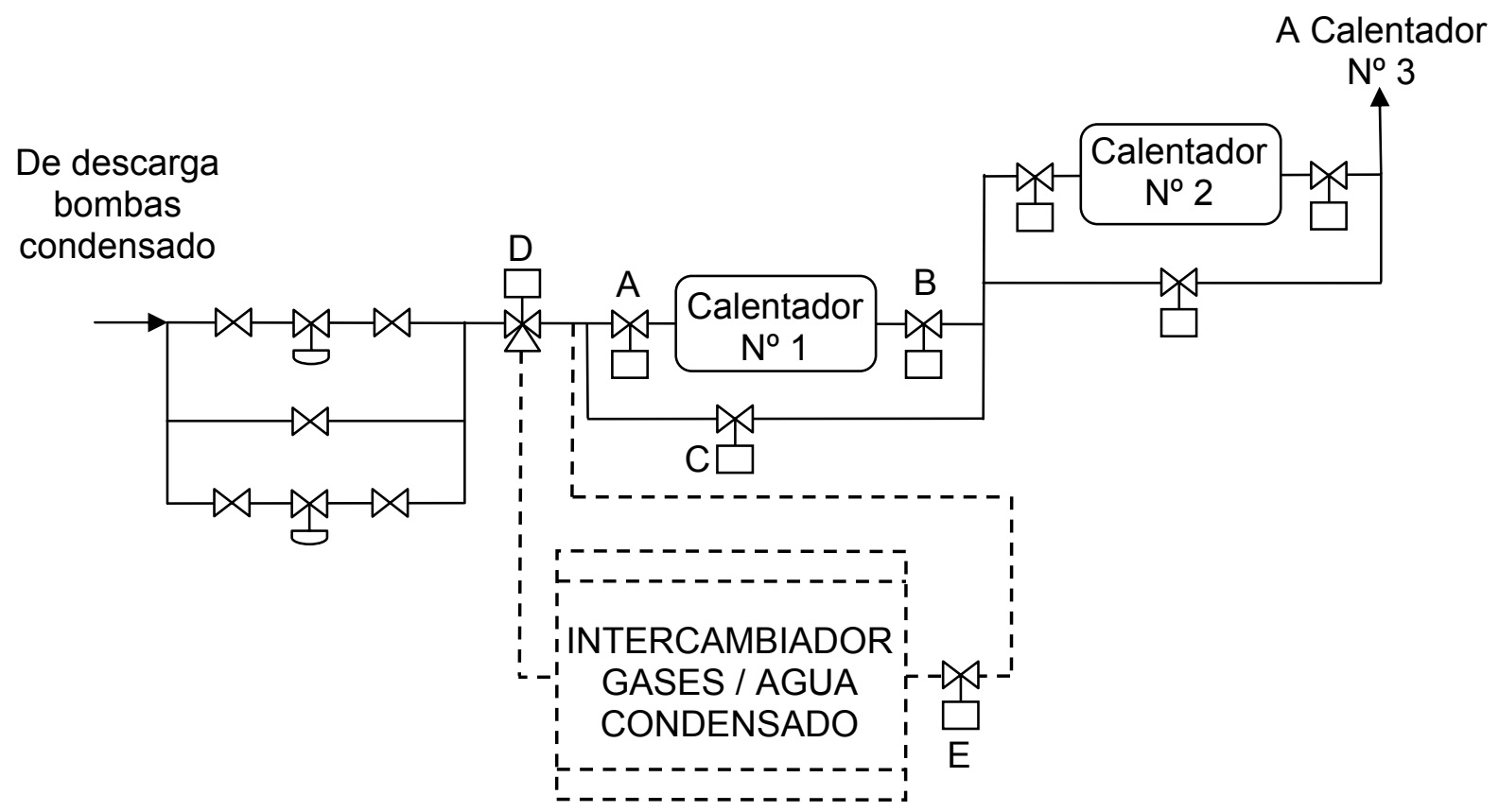

Fig. 1: Modificación propuesta (línea discontinua) para el sistema de condensado. 
En el pozo caliente se almacena el agua condensada procedente del vapor de escape de las secciones de baja presión de turbina, del escape de la turbo bomba y de drenajes. La bomba de condensado aspira el agua del pozo y la impulsa a través de los calentadores descargando en el calentador de mezcla (desaireador) del cual aspiran las bombas de alimentación. Antes de las válvulas motorizadas de entrada $(A)$, salida $(B)$ y by-pass $(C)$ del calentador $N^{\circ} 1$ se instalará una válvula motorizada de 3 vías (D) y a la salida del economizador a condensación una válvula de aislamiento motorizada (E).

Debido a que el posicionamiento de la válvula motorizada de 3 vías permite una regulación del flujo $(0-100 \%)$, el intercambio de calor en el economizador a condensación permitirá una regulación en la misma proporción. El incremento de temperatura de salida del agua de condensado podrá regularse entre dichos márgenes de forma que una temperatura de salida de $80{ }^{\circ} \mathrm{C}$ supondría la anulación total de condensación en el calentador $\mathrm{N}^{0} 1$, mientras que temperaturas intermedias $\left(38-80^{\circ} \mathrm{C}\right)$, supondrían una anulación proporcional de su capacidad de condensación. Asimismo si la temperatura de salida fuera superior a los citados $80{ }^{\circ} \mathrm{C}$, se produciría de forma automática la anulación proporcional de la capacidad de condensación del calentador $\mathrm{N}^{\circ} 2$ y así sucesivamente ocurriría con los siguientes $\left(\mathrm{N}^{\circ} 3, \ldots\right)$ si la temperatura siguiese aumentando.

\section{RESULTADOS Y DISCUSIÓN}

A fin de evitar problemas de penacho a la salida de la chimenea se han considerado dos posibles soluciones, aplicándolas tanto a la central de fuel-oil reconvertida a gas natural como a una central originariamente diseñada para gas natural respectivamente que se resumen a continuación:

a) Utilizar el $100 \%$ de los gases de escape en un economizador a condensación, con la consecuente disminución de temperatura (por debajo de la temperatura de rocío físico) y proceder a un posterior calentamiento por ejemplo con calentadores eléctricos que eviten la formación de penacho. Si no fuese necesario evitar la formación de penacho estos calentamientos no serían necesarios.

b) Utilizar un porcentaje del $63 \%$ de los gases de escape en un economizador a condensación de forma que, posteriormente, al mezclar los gases desviados al economizador con los que pasan a chimenea sin intercambio de calor se obtenga la temperatura en la base de chimenea necesaria para evitar la formación de penacho que es requisito imprescindible según la legislación local a fin de evitar el impacto visual que supone; para ello se ha pensado utilizar unos dampers para regular el flujo y producir las mismas pérdidas de carga.

\section{Central de Fuel-oil reconvertida a gas natural}

Para la central reconvertida a gas natural, se proponen cuatro alternativas, tres de ellas correspondientes a la primera solución y una a la segunda:

Alternativa A: Utilizar la totalidad de los gases en un economizador a condensación para eliminar totalmente la capacidad de condensación del calentador $\mathrm{N}^{\circ} 1$ y la mitad de la capacidad del $\mathrm{N}^{\circ} 2$, aportando $16.233,78 \mathrm{~kW}$ para así evitar la formación del penacho, alcanzando $92^{\circ} \mathrm{C}$ en la base de la chimenea. Los resultados obtenidos son:

$\Delta \eta(P C S)=0,63 \% ; \Delta P=6.692 \mathrm{~kW}$.

Alternativa B: Utilizar la totalidad de los gases en un economizador a condensación para eliminar totalmente la capacidad de condensación del calentador $\mathrm{N}^{\circ} 1$ y la cuarta parte de la capacidad del $\mathrm{N}^{\circ}$ 2, aportando $15.970,47 \mathrm{~kW}$, para así evitar la formación del penacho. Los resultados obtenidos son:

$\Delta \eta(\mathrm{PCS})=0,54 \% ; \Delta \mathrm{P}=5.751 \mathrm{~kW}$ 
Alternativa C: Utilizar la totalidad de gases en un economizador a condensación para eliminar totalmente la capacidad de condensación del calentador $\mathrm{N}^{\circ} 1$, aportando $15.718,38 \mathrm{~kW}$, para así evitar la formación del penacho. Los resultados obtenidos son:

$\Delta \eta(\mathrm{PCS})=0,48 \% ; \Delta \mathrm{P}=5.182 \mathrm{~kW}$.

Alternativa D: Utilizar el $63 \%$ de los gases en un economizador a condensación para eliminar totalmente la capacidad de condensación del calentador $\mathrm{N}^{0} 1$, no necesitándose aportación de calor adicional para evitar la formación de penacho. Los resultados obtenidos son:

$\Delta \eta(P C S)=0,45 \% ; \Delta P=4.770 \mathrm{~kW}$.

Central originariamente diseñada para gas natural.

Para la central de gas natural, se propone sólo una alternativa para cada una de las dos soluciones ya que la temperatura de salida de los gases de combustión no permite mayores aprovechamientos:

Alternativa E: Utilizar la totalidad de los gases en un economizador a condensación para eliminar totalmente la capacidad de condensación del calentador $\mathrm{N}^{\circ} 1$, aportando $16.158,76 \mathrm{~kW}$, para así evitar la formación del penacho. Los resultados obtenidos son:

$\Delta \eta(P C S)=0,44 \% ; \Delta P=4.770 \mathrm{~kW}$.

Alternativa F: Utilizar el $63 \%$ de los gases en un economizador a condensación para eliminar parte de la capacidad de condensación del calentador $N^{0} 1$ no necesitándose aportación de calor adicional para evitar la formación de penacho. Los resultados obtenidos son:

$\Delta \eta(P C S)=0,30 \% ; \Delta P=3.121 \mathrm{~kW}$.

Por una parte, para la central de fuel-oil reconvertida a gas natural, las tres primeras soluciones suponen una complicación adicional en la instalación al tratar de evitar el penacho aunque desde el punto de vista energético resultan más eficientes. Por otra parte, los flujos de aire de la combustión son similares en ambos tipos de centrales porque en aquellas diseñadas originariamente a gas natural hay un fuerte exceso de aire a fin de proteger el recalentador mientras que en las centrales reconvertidas a gas natural, el exceso de aire es limitado por criterios de estabilidad de llama y la protección del recalentador se realiza mediante aportación de agua de atemperamiento.

Los resultados completos más representativos obtenidos en las dos posibles soluciones para cada uno de los dos tipos de centrales, se muestran a continuación en la tabla 2 . En el caso de la central reconvertida utilizando el $100 \%$ de los gases, se ha mostrado la mejor de las alternativas propuestas que corresponde con la primera de ellas.

Tabla 2: Resultados más representativos obtenidos para las dos posibles soluciones.

\begin{tabular}{|l|c|c|c|c|}
\hline & \multicolumn{2}{|c|}{ Central reconvertida } & \multicolumn{2}{c|}{ Central de gas natural } \\
\cline { 2 - 5 } & $100 \%$ & $63 \%$ & $100 \%$ & $63 \%$ \\
\hline Potencia térmica a aportar a los gases $(\mathrm{kW})$ & $16.233,78$ & 0 & $16.158,76$ & 0 \\
\hline Temperatura salida agua del economizador $\left({ }^{\circ} \mathrm{C}\right)$ & 90 & 77 & 77 & 65 \\
\hline Temperatura saturac. gases entrada chimenea $\left({ }^{\circ} \mathrm{C}\right)$ & 54,97 & 54,71 & 55,14 & 54,52 \\
\hline Temperatura en la base de chimenea $\left({ }^{\circ} \mathrm{C}\right)$ & 92 & 88,81 & 92 & 92,6 \\
\hline$\Delta \mathrm{P}(\mathrm{kW})$ & 6.692 & 4.770 & 4.770 & 3.121 \\
\hline$\Delta \eta(\%)$ & 0,63 & 0,45 & 0,44 & 0,30 \\
\hline
\end{tabular}


El incremento porcentual de la eficiencia obtenida es con respecto al poder calorífico superior (PCS) del gas natural quemado en ambas centrales, cuyo valor es de 51.661,95 kJ/kg., siendo la composición volumétrica porcentual obtenida por el cromatógrafo de la estación de control y medida:

$\mathrm{CH}_{4}(88,9786), \mathrm{C}_{2} \mathrm{H}_{6}(5,1274), \mathrm{C}_{3} \mathrm{H}_{8}(1,2714), \mathrm{C}_{4} \mathrm{H}_{10}(0,5573), \mathrm{C}_{5} \mathrm{H}_{12}(0,1451), \mathrm{C}_{6} \mathrm{H}_{14}(0,1200), \mathrm{N}_{2}$ $(3,4898)$ y $\mathrm{CO}_{2}(0,3112)$.

\section{VIABILIDAD DE LAS SOLUCIONES}

El "economizador a condensación" introducido es un intercambiador convencional de carcasa y tubos aleteados de acero al carbono en la zona donde no existe condensación del vapor de agua de los productos de la combustión y de acero inoxidable en la zona donde existe condensación (se podría pensar el la posibilidad de utilizar aluminio para abaratar costes). A la hora de llevar a cabo el estudio de viabilidad se ha considerados el coste total del equipo $(\mathrm{Cl})$ : tubos, codos, colectores de entrada y salida de agua, piezas de apoyo anterior y posterior de los tubos, así como las intermedias, juntas, forros de chapa, bridas de acoplamiento, tolvas (de acero al carbono la de entrada y de acero inoxidable la de salida), estructuras de soporte, etc. (Peña y Blanco, 2006).

La viabilidad de la modificación propuesta se puede considerar desde dos puntos de vista; como un ahorro de combustible para dar la misma potencia o como un aumento en la producción de energía eléctrica, con el consiguiente beneficio. Para el estudio de viabilidad se ha tenido en cuenta los ahorros debidos a la disminución del combustible utilizado (AC) para dar la misma potencia así como los ingresos percibidos por disponibilidad debidos al aumento de la potencia (AR) de la central, valorados en 0,0024 $€ / \mathrm{kWh}$ (Blanco et al., 2006), siendo el ahorro total (AT) la suma de los dos términos anteriores.

El período de retorno simple (RS) de la inversión realizada, asociada principalmente al economizador incluyendo también el coste de tubos y accesorios como se ha comentado antes, se define como:

$\mathrm{RS}=\mathrm{Cl} / \mathrm{AT}$

Además depende en gran medida, de las horas anuales de funcionamiento de la central. Los resultados obtenidos se muestran en las tablas 3 y 4 correspondientes a una central de fuel oil reconvertida a gas natural y una central diseñada originalmente para gas natural respectivamente en las que se consideran 2.000 y $5.000 \mathrm{~h}$. de funcionamiento anuales respectivamente para cada una de las alternativas propuestas.

Tabla 3. Viabilidad de una central de fuel oil reconvertida a gas natural (en base al PCS).

\begin{tabular}{|c|c|c|c|c|c|c|c|c|}
\hline $\begin{array}{c}\text { Alternativa } \\
\text { propuesta }\end{array}$ & $\begin{array}{c}\mathrm{Cl} \\
(\mathrm{M} €)\end{array}$ & $\begin{array}{c}\mathrm{AR} \\
(\mathrm{M} € / \text { año })\end{array}$ & $\begin{array}{c}\mathrm{AC}_{2.000 \mathrm{~h}} \\
(\mathrm{M} € / \mathrm{año})\end{array}$ & $\begin{array}{c}\mathrm{AC}_{5.000 \mathrm{~h}} \\
(\mathrm{M} / \mathrm{año})\end{array}$ & $\begin{array}{c}\mathrm{AT}_{2000 \mathrm{~h}} \\
(\mathrm{M} € / \text { año })\end{array}$ & $\begin{array}{c}\mathrm{AT}_{5.000 \mathrm{~h}} \\
(\mathrm{M} € / \text { año) }\end{array}$ & $\begin{array}{c}\mathrm{RS}_{2.000 \mathrm{~h}} \\
\text { (años) }\end{array}$ & $\begin{array}{c}\mathrm{RS}_{5.000 \mathrm{~h}} \\
\text { (años) }\end{array}$ \\
\hline $\mathrm{A}$ & 1,727 & 0,141 & 0,648 & 1,622 & 0,789 & 1,763 & 2,19 & 0,98 \\
\hline $\mathrm{B}$ & 1,063 & 0,121 & 0,557 & 1,393 & 0,678 & 1,514 & 1,57 & 0,70 \\
\hline $\mathrm{C}$ & 0,865 & 0,109 & 0,502 & 1,255 & 0,611 & 1,364 & 1,42 & 0,63 \\
\hline $\mathrm{D}$ & 1,164 & 0,100 & 0,470 & 1,175 & 0,570 & 1,275 & 2,04 & 0,91 \\
\hline
\end{tabular}

Tabla 4. Viabilidad de una central original de gas natural (en base al PCS).

\begin{tabular}{|c|c|c|c|c|c|c|c|c|}
\hline $\begin{array}{l}\text { Alternativa } \\
\text { propuesta }\end{array}$ & $\begin{array}{c}\mathrm{Cl} \\
(\mathrm{M} €)\end{array}$ & $\begin{array}{c}\mathrm{AR} \\
\text { (M€/año) }\end{array}$ & $\begin{array}{l}\mathrm{AC}_{2.000 \mathrm{~h}} \\
\text { (M€/año) }\end{array}$ & $\begin{array}{c}\mathrm{AC}_{5.000 \mathrm{~h}} \\
\text { (M€/año) }\end{array}$ & $\begin{array}{c}\mathrm{AT}_{2.000 \mathrm{~h}} \\
\text { (M€/año) }\end{array}$ & $\begin{array}{c}\mathrm{AT}_{5.000 \mathrm{~h}} \\
\text { (M€/año) }\end{array}$ & $\begin{array}{c}\mathrm{RS}_{2.000 \mathrm{~h}} \\
\text { (años) }\end{array}$ & $\begin{array}{l}\mathrm{RS}_{5.000 \mathrm{~h}} \\
\text { (años) }\end{array}$ \\
\hline$E$ & 1,172 & 0,100 & 0,445 & 1,111 & 0,545 & 1,211 & 2,15 & 0,96 \\
\hline$F$ & 0,912 & 0,066 & 0,294 & 0,735 & 0,360 & 0,801 & 2,53 & 1,14 \\
\hline
\end{tabular}




\section{CONCLUSIONES}

A partir de los resultados obtenidos en el presente estudio, es posible concluir lo siguiente:

El aprovechamiento del calor de los gases de la combustión, especialmente del calor latente de una parte del vapor de agua contenido en los mismos, puede elevar el rendimiento térmico de las centrales termoeléctricas convencionales que funcionan con gas natural así como lograr un aumento en la potencia. En nuestro caso para unas centrales tipo estudiadas de $379 \mathrm{MW}$ reconvertidas a gas natural, se conseguirían incrementos del orden de 0,60\% en la eficiencia energética global con respecto al PCS, lo cual supondría un incremento de algo más de $6.600 \mathrm{~kW}$ en la potencia (Alternativa A). Para el caso de centrales originalmente diseñadas para quemar gas natural, los incrementos obtenidos han sido sensiblemente inferiores, del orden de $0,44 \%$ en la eficiencia energética global también con respecto al PCS, lo cual supondría un incremento de algo más de $4.770 \mathrm{~kW}$ en la potencia (Alternativa E), debido principalmente a que la temperatura de salida de gases tras los calentadores es del orden de $120^{\circ} \mathrm{C}$ mientras que en las de fuel oil reconvertidas a gas natural es del orden de $147^{\circ} \mathrm{C}$.

En cuanto al tipo de solución adoptada, el incremento de la eficiencia global de las alternativas que utilizan el $100 \%$ de los gases de la combustión (Alternativas $A, B, C$ y E) es mayor que en los casos del $63 \%$ (Alternativas D y F), debido a que el calor latente se aprovecha en su totalidad en la primera solución mientras que en la segunda, una parte del mismo se desperdicia en el condensador.

En cuanto al análisis económico para ambos tipos de centrales, se han logrado períodos de retorno simple de la inversión del orden de 2 años funcionando a un régimen de $2.000 \mathrm{~h} / a n ̃ o$ mientras que con un incremento del régimen de trabajo a $5.000 \mathrm{~h} /$ año los períodos de retorno disminuirían a alrededor de 1 año, los cuales resultan aceptables en este tipo de instalaciones para unas inversiones razonables.

El único objetivo de los calentamientos adicionales propuestos en este estudio mediante calentadores eléctricos es el de evitar el penacho (según normativas locales de obligado cumplimiento), de forma que allí donde no fuese obligatorio, se podría prescindir de los mismos por lo que la eficiencia energética aumentaría y los períodos de retorno disminuirían con respecto a los obtenidos en este estudio.

El sistema propuesto en este estudio es autorregulado ya que a medida que se va calentando el agua, el salto térmico entre el agua aportada al calentador y el vapor de la extracción es menor, de forma que si no hubiera salto térmico no habría condensación, luego es el propio sistema el que se autorregula dependiendo de las condiciones de operación.

Además, es completamente flexible, ya que permite su retirada de servicio de forma inmediata, en y durante cualquier modo de operación, con lo que si se desea se puede descartar la modificación propuesta y adecuarse a su condición original de partida en todo momento.

Por último, cabe señalar que en el caso de los combustibles fósiles tradicionales como el fuel-oil, la aplicación de esta metodología puede verse limitada por dos motivos principalmente; Por una parte la presencia excesiva de azufre en la matriz del combustible con los problemas tecnológicos derivados y por otra el nuevo contenido de vapor de agua en los productos de la combustión con la consiguiente disminución de la temperatura de rocío física.

En el caso de la biomasa tan de moda en la actualidad desde el punto de vista medioambiental, el problema vendría a ser la composición de los gases de la combustión, pero el aprovechamiento según esta metodología podría ser de gran interés, dada la gran cantidad de vapor de agua que en muchos casos hay contenida en dichos gases. 


\section{NOMENCLATURA}

$\begin{array}{ll}\text { AC } & \text { Ahorro en Combustible (M€/año) } \\ \text { AR } & \text { Ahorro en Repotenciación (M€/año) } \\ \text { AT } & \text { Ahorro Total (M€/año) } \\ \Delta & \text { Incremento } \\ \mathrm{Cl} & \text { Coste de la inversión }(\mathrm{M} €) \\ \mathrm{M} € & \text { Millones de euros } \\ \eta & \text { Eficiencia global }(\%) \\ \mathrm{P} & \text { Potencia total }(\mathrm{kW}) \\ \mathrm{PCS} & \text { Poder Calorífico Superior }(\mathrm{kJ} / \mathrm{kg}) \\ \mathrm{RS} & \text { Período de Retorno Simple }(\mathrm{años})\end{array}$

\section{REFERENCIAS}

Bastidas, M.J., Bermúdez, R.F., Jaramillo, G.P y Chejne, F., Optimización termo económica y ambiental usando algoritmos genéticos multiobjetivo. Información Tecnológica, 21, 4, 35-44, (2010).

Blanco, J.M. y Peña, F., Obtención del valor real de las pérdidas de difícil evaluación aplicables al cálculo de rendimiento de calderas, Información Tecnológica, 17, 3, 123-128, (2006).

Blanco, J.M., Mendía, F. y Peña, F., Comparative analysis of $\mathrm{CO}_{2}$ and $\mathrm{SO}_{2}$ emissions between combined and conventional cycles with natural gas and fuel oil consumption over the Spanish thermal power plants, Fuel, 85, 1280-1285, (2006).

Chacón, J., Sala, J.M. y Blanco, J.M., Investigation on the Design and Optimization of a Low NOxCO Emission Burner both experimentally and through CFD simulations, Energy and Fuels, 21, 4258, (2006).

Chen, M., Lund, H., Rosendahl, L.A. y Condra, T.J., Energy efficiency analysis and impact evaluation of the application of thermoelectric power cycle to today's CHP systems, Applied Energy, 87, 1231-1238, (2010).

Demirbas, A., The sustainability of combustible renewables, Energy Sources, Part A, 30, 11141119, (2008).

Grohn, A., Suonmaa, V., Auvinen, A., Lehtinen, K.E.J. y Jokiniemi, J. Reduction of fine particle emissions from wood combustion with optimized condensing heat exchangers. Environ. Sci. Technol., 43, 6269-6274, (2009).

Guerra, S.G., Vázquez-Román, R. y Rodríguez-Toral, M.A. Simulación de Plantas de Cogeneración de Ciclo Combinado usando ASPEN®. Información Tecnológica, 16, 1, 42-49, (2005).

Hongbin, Z. y Pengxiu, Y., Study of humid air turbine cycle with external heat source for air humidification, International Journal of Energy Research, 34, 6, 523-534, (2010).

Ion, V.I., Popescu, F. y Georgescu, L., Prediction of the pollutants generation in natural gas/residual steel gases co-combustion, Int. J. of energy and environment, 2, 1, 79-84, (2007).

Peña, F. y Blanco, J.M., Evaluation of the physical dew point in the economizer of a combined cycle burning natural gas, Applied Thermal Engineering, 27, 2153-2158, (2007).

Remiro, J.A. y Lozano, M.A., Control del Rendimiento y Diagnóstico Termoeconómico de Centrales Termoeléctricas. Información Tecnológica, 18, 1, 87-96, (2007).

Schneider, D.R. y Bogdan, Ž., Effect of heavy fuel oil/natural gas co-combustion on pollutant generation in retrofitted power plant, Applied Thermal Engineering, 27, 1944-1950, (2007). 